\title{
Aspects of follicular and oocyte maturation that affect the developmental potential of embryos
}

\author{
P. Mermillod, B. Oussaid and Y. Cognié \\ Instifut National de la Recherche Agronomique, Station de Physiologie de la Reproduction des \\ Mammiferes Domestiques, 37380 Nouzilly, France
}

The ability to mature, be fertilized and finally to develop into a viable embryo is acquired gradually by the oocyte during progressive differentiation throughout folliculogenesis. This process starts with oocyte growth during the first steps of follicular development. As the oocyte is close to its final size, other modifications occur, less spectacular but at least as important in determining the resulting ability of the oocyte to accomplish its reproductive purpose (developmental competence). These modifications, referred to as 'oocyte capacitation', are probably influenced by the follicle. The proportion of developmentally competent oocytes increases with follicular size. However, the relationship between follicular growth and oocyte competence is not very strict, since a given oocyte may acquire its competence at any stage of follicular growth and since some examples of functional disjunction between follicular size and oocyte competence are described. Follicular atresia may impair the acquisition of oocyte competence, as evidenced by the parallel study of follicular characteristics and of the developmental potential of their oocytes treated individually through in vitro maturation, fertilization and development. However, when atresia is experimentally induced in large preovulatory follicles, oocytes remain competent, indicating that once competence is acquired, it is no longer sensitive to atresia. Oocyte maturation represents only the end of this long and progressive process and validates the preparation of the oocyte by conferring its final developmental ability. As evidenced by recent cloning experiments, the cytoplasmic aspects of oocyte maturation are crucial for the acquisition of developmental competence. This cytoplasmic maturation may be activated in vitro by the use of complex media supplement (serum, follicular fluid) but the use of defined media for maturation allowed the identification of some active factors (such as epidermal growth factor, growth hormone, inhibin and activin). The study of some differential models of oocyte competence (follicular size and atresia, Booroola gene, prepubertal oocytes) will provide a better understanding of oocyte capacitation and maturation, and allow the improvement of in vitro methods for oocyte maturation, which represent the most limiting step of in vitro production of embryos in large mammals.

\section{Introduction}

Despite the vast amount of work during the last twenty years aimed at improving in vitro production (IVP) of embryos in domestic species, the percentages of in vitro matured (IVM), in vitro fertilized (IVF) oocytes reaching the blastocyst stage after in vitro development (IVD) still reach a plateau at $30-40 \%$, In vitro development has been the subject of many studies in recent years and many advances have been made. The use of oviductal cells in coculture first allowed the speciesspecific block of development to be overcome and produced the first success in IVP. Semi-defined and fully defined embryo culture systems have been devised and used with equal or better success rates than coculture (see Thompson, 1996 for review). After much investigation of embryo culture 
systems, it now seems more likely that oocyte maturation is the more limiting step in the in vitro production of embryos. The second limitation of the use of IVP concerns embryo viability after transfer, especially with frozen-thawed embryos. It appears that the origin of this limitation is in the system used for embryo development (reviews by Massip et al., 1995; Thompson, 1997).

There are differences between oocytes in their ability to be fertilized and to develop to the blastocyst stage in any IVF-IVD system. This ability has been referred to as oocyte quality or oocyte developmental competence. The competence of a mature oocyte depends on at least two factors: (1) oocyte capacitation, that is the preparation of the oocyte during folliculogenesis and especially during the later phases of follicular growth (Hyttel et al., 1997), and (2) the morphological and biochemical modifications of oocytes taking place during maturation, after the LH surge or after removal of oocytes from the inhibitory effects of the follicle. These two aspects of oocyte quality do not have the same consequences for IVP. It is possible to adapt IVM conditions to improve the quality of oocyte maturation and several advances have been made during the last decade. However, improving oocyte capacitation is more difficult, because oocytes resume meiosis in vitro and capacitation ceases as soon as they are removed from the follicle. The follicular environment is probably one of the most important parameters regulating capacitation. Until now, no well established culture system for germinal vesicle stage oocytes has been available for large domestic animals. Consequently, the improvement of the quality of oocytes used for IVM depends upon the manipulation of the physiology of the whole animal to increase the number of follicles containing competent oocytes. Much work has been devoted to the isolation and in vitro development of small preantral follicles and some success have been achieved in rodent species (review by Eppig et al., 1996). In larger animals it has been shown that a large number of preantral follicles can be obtained from ovaries (Nuttinck et al., 1993). However, a better understanding of oocyte differentiation and interactions between oocyte and follicular cells is required before efficient systems can be set up to produce fully grown and competent oocytes from preantral or small antral follicles, even though some encouraging results have been obtained by Harada et al. (1997).

The first step in the acquisition of developmental competence is oocyte growth at the start of follicular development. This important differentiation process will not be considered here, but good reviews of mechanisms and regulations involved include those by Eppig et al. (1996), Gosden et al. (1997) and Hyttel et al. (1997).

In this review follicular factors that affect the capacitation and maturation of oocytes and their resulting ability to develop into viable embryos are discussed.

\section{Oocyte Capacitation}

Acquisition of oocyte developmental competence occurs continuously throughout folliculogenesis (Fig. 1). This acquisition can be divided in three separate stages defined by particular physiological events: (1) oocyte growth, which takes place mainly during the beginning of follicle emergence (primary and secondary preantral follicles); (2) oocyte capacitation, starting at the end of oocyte growth in antral (tertiary) follicles; and (3) oocyte maturation, starting after the LH surge in preovulatory follicles or after removal of the oocyte from the follicular environment which inhibits meiotic resumption.

Among a batch of oocytes collected from small to medium size follicles of cattle, sheep or goat ovaries at an abbatoir, more than $90 \%$ will reach metaphase II of meiosis after IVM; more than $70 \%$ will be successfully fertilized (two pronuclei after IVF) and cleave; but only a third will develop to the morula-blastocyst stage after IVD (Fig. 2). This finding indicates that among a batch of oocytes, a large number are unable to develop normally beyond the first cleavage. This is a common observation in different species and in different IVP systems (see review by Mermillod et al., 1996). The explanation for this phenomenon becomes apparent when it is considered that oocyte developmental competence is continuously increasing during folliculogenesis and is also regulated by other characteristics of follicular physiology: oocytes collected from the heterogeneous follicular population of ovaries from an abattoir are of different developmental ability. 


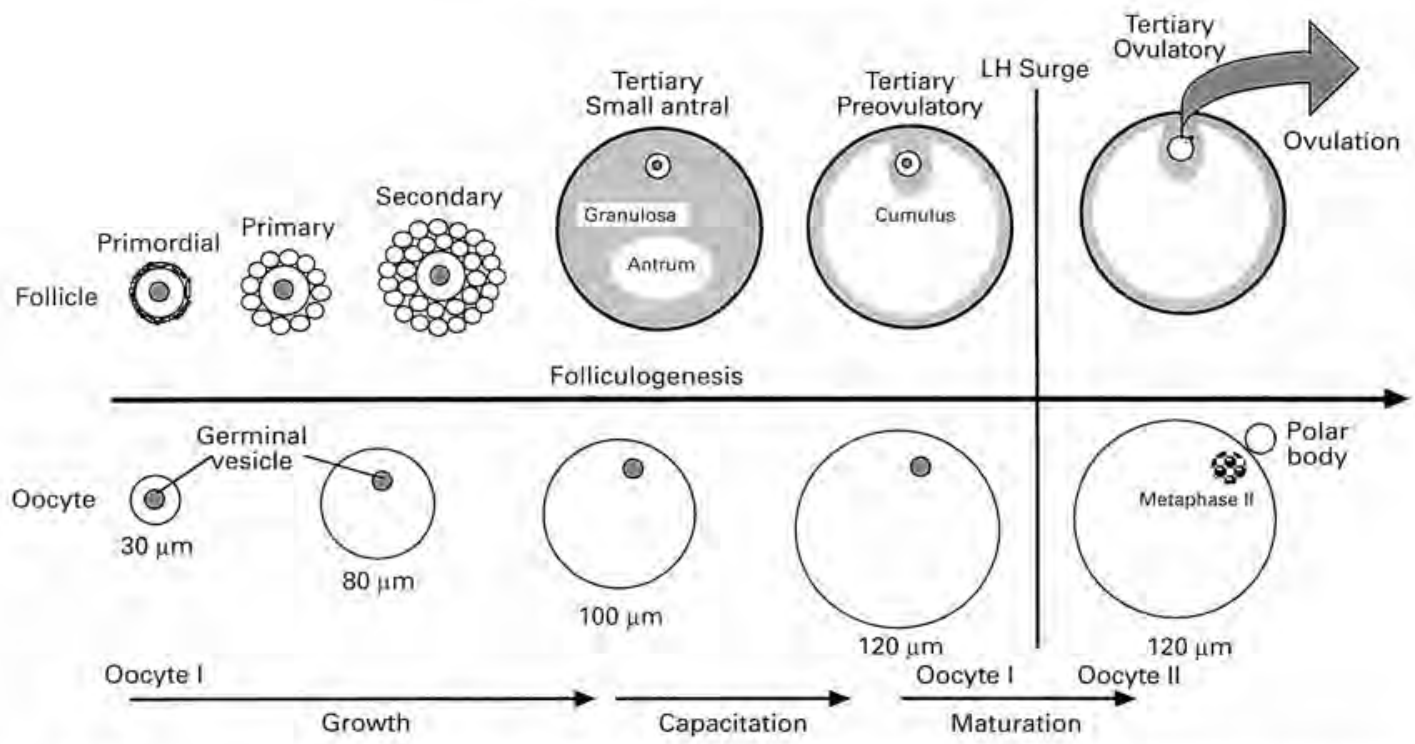

Fig. 1. Schematic representation of oocyte growth, capacitation and maturation along the axis of folliculogenesis. Oocyte growth is initiated as soon as the follicle enters the pool of growing follicles and is almost completed upon antrum formation even if some increase in size is observed up to ovulation. Other modifications take place in an antral follicle during oocyte capacitation, conferring developmental competence to the oocyte. Maturation, after the LH surge or removal of oocyte from the inhibitory environment of the follicle, is necessary to prepare the haploid chromosome complement of oocyte II and to express the developmental potential acquired during capacitation.

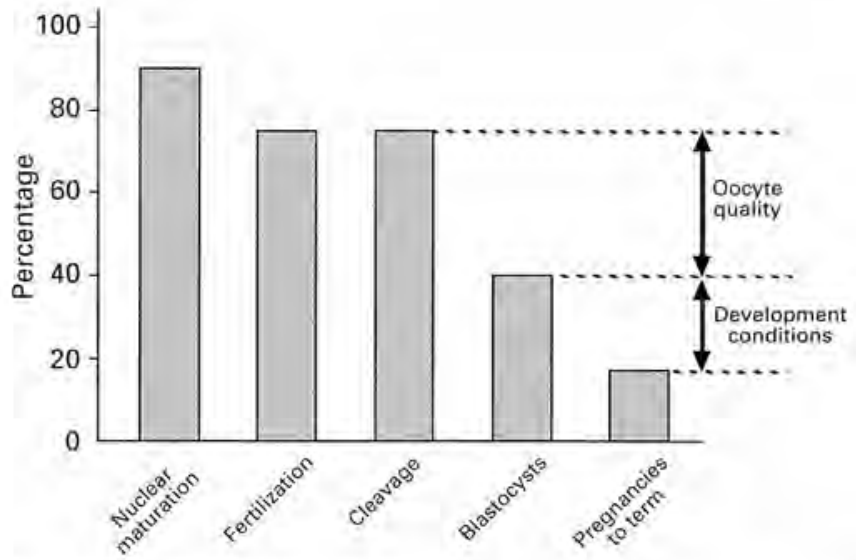

Fig. 2. Successive steps of embryo production in vitro. Meiotic maturation occurs at very high rates as do fertilization and cleavage of early embryos in three domestic ruminant species (cattle, sheep and goat). The first decrease in success is observed at the blastocyst stage and may be attributed to the low oocyte capacitation or suboptimal conditions used for IVM. A second decrease is observed in the pregnancy rate obtained after transfer of in vitro produced embryos (particularly after a cryopreservation step) that could be attributed to the conditions used for embryo development. 


\section{Follicle size}

The first indication of the presence of oocyte capacitation was obtained by evaluating the developmental potential of immature oocytes collected from follicles of known size (cattle: Pavlok et al., 1992; Lonergan et al., 1994a; goat: Crozet et al., 1995; and sheep: Cognié et al. 1998). When follicles are dissected and classified according to their size (size classes are different between species), the oocytes harvested from larger follicles provide better development results than those from smaller follicles. Consequently, large follicles contain a higher proportion of developmentally competent oocytes. Since 'blastocyst rate' is measured at the end of IVP and some blastocysts are observed even when oocytes are harvested from smaller follicles, it is more likely that oocyte capacitation is occurring with different kinetics between follicles. If we consider the smaller follicles, only a few oocytes appear competent (the faster oocytes), whereas in larger follicles, even more slowly developing oocytes have had time to complete capacitation.

Thus, the question is: what determines the kinetics of oocyte capacitation during folliculogenesis? Capacitation could be regulated by the oocyte itself or by follicular characteristics other than size. In this view, it is of interest to note that for an equal follicular size, oocytes from peripheral follicles (follicles visible at the surface of the ovary) are larger and have a higher meiotic and developmental competence than oocytes from follicles localized in the ovarian cortex (Arlotto et al., 1996). This result indicates that the timing of capacitation of an oocyte could differ in accordance with the location of its follicle.

Another example of functional disjunction between follicular growth and acquisition of meiotic and developmental ability by the oocyte is found in ewes bearing the Booroola fecundity gene (Bindon, 1984). It has been shown that in heterozygous ewes $\left(\mathrm{Fec}^{\mathrm{B}} \mathrm{Fec}^{+}\right)$, the rate of development of oocytes from a given follicular size class is superior to the rate observed for oocytes obtained from the same size class in females of wild $\left(\mathrm{Fec}^{+} \mathrm{Fec}^{+}\right.$) genotype (Cognié et al., 1998). This difference in the timing of acquisition of competence is reflected earlier since, even in preantral follicles, oocytes from ewes bearing the Booroola gene are larger than oocytes from wildtype animals at the same follicular stage. As a consequence, a single gene may be able to regulate the kinetics of the capacitation of the oocyte inside the growing follicle. The identification of this gene will improve understanding of oocyte capacitation.

Considering these facts, it is clear that some important but unknown events leading to acquisition of developmental competence take place in oocytes at variable stages of follicular development. Oocytes that do not complete this preparation are not competent to develop even if they are already able to resume meiosis and to be fertilized. The morphological and biochemical basis of this capacitation remains to be determined; however, it probably involves the transcription of some genes important for survival or for the regulation of gene expression in early embryos. The reconstitution in vitro of this capacitation step will require the control of meiotic inhibition to allow the oocyte to complete transcription of necessary genes before chromosome condensation. Knowledge of the mechanisms of meiotic inhibition is not yet sufficient for the establishment of culture systems for germinal vesicle stage oocytes even though some encouraging results have been obtained (Lonergan et al., 1997; Van Tol et al., 1996).

To summarize, it appears that the proportion of competent oocytes increases with follicular size. Oocyte capacitation (that is, the ability to accomplish the cytoplasmic aspects of maturation successfully) may be acquired by the oocyte at any time during growth of antral follicles. The mechanisms and the follicular regulation of capacitation are unknown.

\section{Follicular atresia}

Follicular degeneration, or atresia, is the more probable destiny of any given follicle. A high proportion of follicles present at the surface of ovaries are at various stages of atresia (review by Monniaux et al., 1997). Consequently, it is important to know to what extent atresia could influence the acquisition of developmental competence by the oocyte and to determine whether oocytes from atretic follicles could be rescued during in vitro culture when they would have been lost normally during normal degeneration processes in vivo (review by Sirard and Blondin, 1996). 
The main problem encountered in the study of the effect of atresia on oocyte developmental competence is that no macroscopic criteria are available for selecting and classifying follicles according to their health status. The criteria usually used for the determination of atresia are biochemical: steroid content of the follicular fluid (Grime and Ireland, 1986), or pattern of expression of insulin-like growth factor binding proteins (IGFBP, Monget et al., 1993). Atresia may also be evaluated by the rate of mitosis-pycnosis or apoptosis in granulosa cells (review by Monniaux et al., 1997). All these methods are time consuming and thus do not allow classification and pooling of oocytes before IVM according to the stage of atresia of the follicles they come from. Consequently, the parallel study of atresia and oocyte competence requires the use of IVM, IVF, IVD methods designed for individual oocytes, whereas oocytes are usually treated in groups and cooperation among oocytes or embryos has been reported (Ferry et al., 1994; Blondin and Sirard, 1995). However, it appeared that IVP techniques could be successfully adapted to individual oocytes or embryos (Carolan et al., 1996a) and some results in the comparative study of follicular characteristics, morphology of cumulus-oocyte complexes and oocyte developmental competence using IVM, IVF and IVD systems designed for individual oocytes have been obtained recently in cattle. These results are slightly controversial. For example, in one study (Hazeleger et al., 1995), follicles containing highly competent oocytes were characterized by lower progesterone concentration in follicular fluid, whereas oestradiol was not affected. In a more recent study (Driancourt et al., 1998), the wall of follicles containing oocytes able to develop to the blastocyst stage had a higher aromatase activity compared with follicles containing oocytes unable to develop beyond the 8-16-cell stage (Fig. 3), although the steroid content of follicular fluid was not affected. These authors also reported a higher concentration of inhibin ( $\alpha$ subunit) in the fluid of follicles containing developmentally competent oocytes. This finding is of interest considering the positive effect of inhibin-activin on oocyte maturation (see below) and considering that the concentration of inhibin $\alpha$ subunit is strongly reduced during follicular regression caused by atresia (Guilbault et al., 1993). In conclusion, the clear relationship between follicular atresia and acquisition of developmental competence by an oocyte is not fully established, although some data indicate that competent oocytes are more frequently found in physiologically active follicles. This is not surprising as it is known that developmental competence may be acquired by oocytes at any time during follicular development. The occurrence of atresia before that time could deprive the slower oocytes of any chance of gaining this competence (Fig. 4).

Once an oocyte has reached its full developmental competence, it appears less dependent upon follicular health status. To test this hypothesis, we maintained preovulatory follicles for increasing periods after FSH superovulation of synchronized heifers treated with the GnRH antagonist Antarelix (Gift from Europeptides, Dr Deghenghi, Argenteuil, France) to inhibit the ovulatory surge of LH (Oussaid, Lonergan and Mermillod unpublished data). FSH treatment consisted of a total of $24 \mathrm{mg}$ pFSH (kindly provided by J-F Beckers, University of Liège) injected twice a day for 4 days in a regimen of decreasing doses. Animals received $1 \mathrm{ml}$ Prosolvin (Intervet, Boxmeer) on the last day of FSH treatment. Heifers were divided among three treatments : (1) control group, killed $24 \mathrm{~h}$ after the last FSH injection $(n=4)$; (2) group $36 \mathrm{~h}$, killed $36 \mathrm{~h}$ after the control group and injected with Antarelix every $12 \mathrm{~h}(1.6 \mathrm{mg})$ starting $24 \mathrm{~h}$ after the last FSH injection $(n=4)$; and (3) group $60 \mathrm{~h}$, killed $60 \mathrm{~h}$ after the control group and injected with Antarelix in the same way $(n=4)$. Ovaries were collected at slaughter and all follicles larger than $5 \mathrm{~mm}$ present at their surface were dissected free of surrounding tissues and opened with a scalpel. The oocyte was retrieved; a sample of follicular fluid was collected and centrifuged for measurements of steroids (radioimmunoassay after extraction, Thibier and Saumande, 1975) and some granulosa cells were smeared on to a microscope slide for evaluation of cell viability (mitosis and pycnosis frequencies after Feulgen staining). Oocytes were treated individually through IVM, IVF, IVD protocols previously described for individual oocytes (Carolan et al., 1996a) and the developmental stage reached by each oocyte was scored as well as the number of cells in the resulting embryo (Hoechst fluorescent staining after ethanol fixation). The maintenance of preovulatory follicles was detrimental to follicular health, inducing a high rate of atresia as evidenced by the pycnotic index of granulosal cells (less than $10 \%$ of follicles showing pycnotic bodies in the control group compared with 58 and $90 \%$ in the 36 and $60 \mathrm{~h}$ groups, 


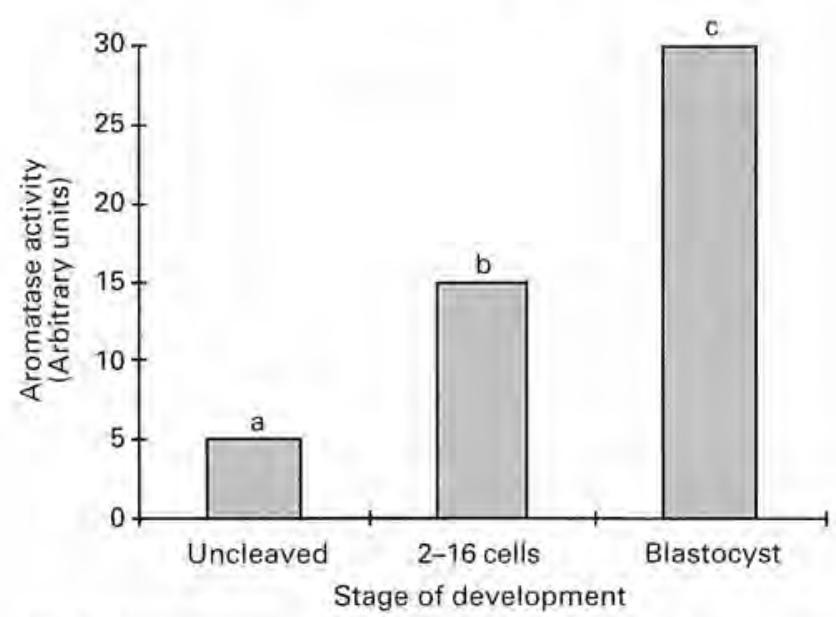

Fig. 3. Aromatase activity in the wall of follicles containing oocytes that are unable to cleave, blocked at early cleavage stage or developing to the blastocyst stage in individual conditions (MA Driancourt, B Thuel, P Mermillod and P Lonergan, unpublished). a,bec Columns with different letters are significantly different ( $P<0.05$, Chi square).

respectively) and a decrease in the concentrations of oestradiol and progesterone. This result was observed after $36 \mathrm{~h}$ and increased after $60 \mathrm{~h}$ (Table 1). However, the developmental potential of the oocytes collected from these advanced atretic follicles was not affected and the quality of the resulting embryos was not affected, as indicated by the blastocyst rate and number of cells (Table 2).

To summarize these observations, it appears that atresia occurring in small-medium size follicles may be detrimental to the developmental potential of the oocytes, at least when physiological characteristics of the follicle are affected (for example aromatase activity). In large preovulatory follicles, where most oocytes are already fully competent, atresia no longer affects competence.

\section{Oocyte Maturation}

The preovulatory LH surge or removal of the oocyte from its follicular inhibitory environment triggers the resumption of meiosis, the most visible feature of oocyte maturation. The meiotic process confers on the secondary oocyte its final haploid DNA complement. Meiosis and its molecular regulation have been studied and described extensively (for reviews see Wassarman and Albertini, 1994; Downs, 1996; Taieb et al., 1997). Beyond these nuclear aspects of oocyte maturation, cytoplasmic events also occur and seem important for the fertilization and development ability of the oocytes (Eppig, 1996). These aspects have been termed cytoplasmic maturation as opposed to the meiotic events called nuclear maturation. Some ultrastructural and biochemical features of cytoplasmic maturation have been described (Wassarman and Albertini, 1994) but the events determining the final quality of the mature oocyte remain to be identified. Recent cloning experiments using fully differentiated donor nuclei have underlined the power of oocyte cytoplasm in reprogramming the nucleus for complete development (Campbell and Wilmut, 1997), Other cloning experiments also indicated the importance of cytoplasmic maturation in determining developmental potential. When enucleated oocytes of different origins are used as recipients for the same batch of embryonic nuclei, they develop at different rates. This has been shown by comparing enucleated oocytes from adult and prepubertal cattle (Mermillod et al., 1998) and from different classes of follicular size (Kubota and Yang, 1998). 


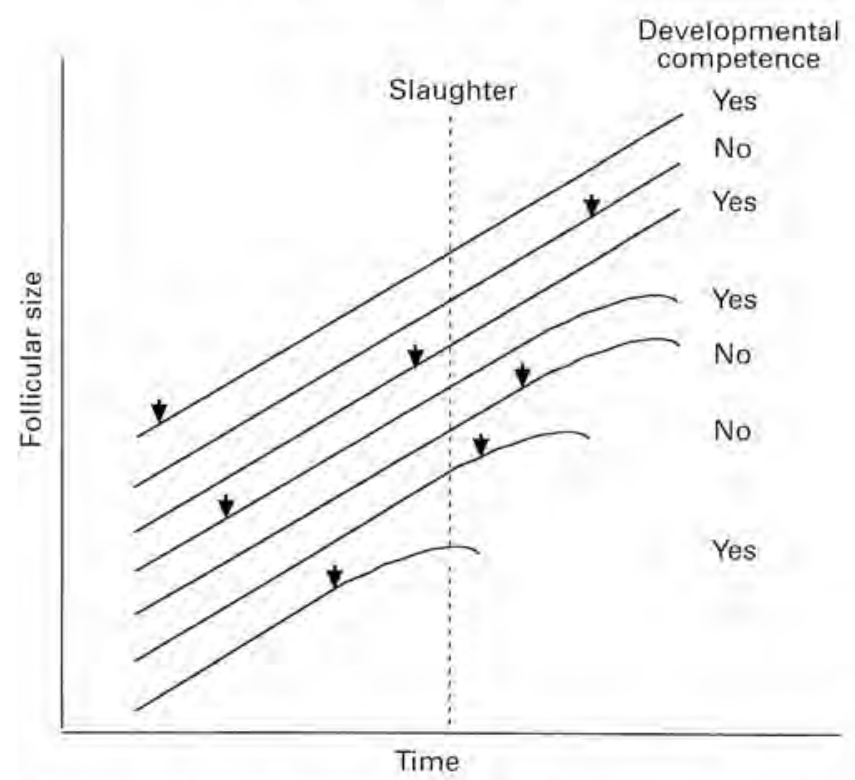

Fig. 4. Hypothetical explanation of the heterogeneity of oocytes collected from a particular ovary. Oocyte developmental competence can appear at any stage of antral folliele development, thus competent oocytes are recovered more frequently from large follicles. As a consequence, some oocytes from large follicles are still incompetent and some oocytes from small follicles are already competent. Atresia develops at any stage of follicular growth. When it appears in large follicles, the oocyte is often already competent and remains competent during the first steps of follicle regression. When atresia occurs in smaller follicles, the oocyte is rarely already competent and hence capacitation is stopped before competence is acquired. Arrows represent acquisition of competence and atresia is represented by inflexion of the curve.

Nuclear maturation occurs spontaneously in vitro in most oocytes and large numbers of mature (metaphase II) oocytes can be obtained in very simple culture conditions (Lonergan et al., 1994b). Even oocytes from small follicles are able to mature properly, and most of them show normal haploid metaphase II after IVM (90\%, follicles of 1-3 mm in diameter in cattle, Ectors et al., 1995). Cytoplasmic maturation, on the other hand, is dependent on conditions used for IVM and could be stimulated by addition of growth factors to the culture medium. In vivo, follicular environment could regulate cytoplasmic maturation as evidenced by experiments of Moor et al. (1996), showing that the first $6 \mathrm{~h}$ spent by the oocyte inside the LH-stimulated follicle in sheep are important for its developmental competence.

Follicular fluid used as a supplement in maturation medium is a potent activator of oocyte maturation, at least in the cytoplasm. This positive effect of follicular fluid seems to be independent of follicular size. However, it seems to be influenced by the stage of atresia (sheep: Cognié et al., 1995; cattle: Carolan et al, 1996b) as well as by the stage of follicular development (growing or regressing follicles, Sirard et al., 1995). Follicular fluid seems thus to contain maturation-activating factors from early stages of follicular growth. The hormonal environment may act by regulating the effect of such factors on the cumulus-oocyte complexes. The molecular effector(s) mediating this positive influence of the follicular environment are not yet fully elucidated. However, immunohistochemical studies of the ovary as well as in vitro maturation experiments have allowed the identification of some factors in follicles (ligand and receptor) and of their effect on in vitro maturation. 
Table 1. Concentrations of oestradiol and progesterone (ng $\mathrm{ml}^{-1} \pm \mathrm{SD}$ ) in follicular fluids of superovulated heifers slaughtered $24 \mathrm{~h}$ after the last FSH injection (control), $36 \mathrm{~h}$ later with injection of $1.6 \mathrm{mg}$ Antarelix every $12 \mathrm{~h}(36 \mathrm{~h})$ or $60 \mathrm{~h}$ later with Antarelix in the same way $(60 \mathrm{~h})$

\begin{tabular}{lcrr}
\hline & \multicolumn{3}{c}{ Treatment } \\
\cline { 2 - 4 } & Control $(n=54)$ & $36 \mathrm{~h}(n=64)$ & $60 \mathrm{~h}(n=52)$ \\
\hline Oestradiol & $788.3 \pm 39.3^{\mathrm{a}}$ & $17.3 \pm 1.2^{\mathrm{b}}$ & $4.0 \pm 0.5^{\mathrm{c}}$ \\
Progesterone & $736.7 \pm 46.2^{\mathrm{a}}$ & $54.8 \pm 3.5^{\mathrm{b}}$ & $54.3 \pm 3.5^{\mathrm{b}}$ \\
\hline
\end{tabular}

$a, b, c$ Values in the same row with different superscripts are significantly different $(P<0.05, t$ test). $n=$ number of follicles obtained from the four heifers in each group.

Table 2. Development of bovine oocytes after individual IVM/IVF/IVC of oocytes recovered from superovulated heifers killed $24 \mathrm{~h}$ after the last FSH injection (control) or maintained for a further 36 or $60 \mathrm{~h}$ with administration of Antarelix to inhibit the LH surge before slaughter

\begin{tabular}{|c|c|c|c|c|c|}
\hline \multirow[b]{2}{*}{ Treatment } & \multirow[b]{2}{*}{$n$} & \multirow{2}{*}{$\begin{array}{c}\text { Cleavage } \\
\text { at } 72 \mathrm{~h} \mathrm{pi} \\
n(\%)\end{array}$} & \multicolumn{2}{|c|}{ Development 8 days pi } & \multirow[b]{2}{*}{$\begin{array}{l}\text { Cell number } \\
x \pm \text { SEM }\end{array}$} \\
\hline & & & $\begin{array}{c}\text { Morula- } \\
\text { blastocysts(\%) }\end{array}$ & Blastocysts (\%) & \\
\hline Control & 54 & $46(85)$ & $22(41)^{\mathrm{a}}$ & $22(41)$ & $95 \pm 8$ \\
\hline $36 \mathrm{~h}$ & 64 & $55(86)$ & $39(61)^{b}$ & $36(56)$ & $93 \pm 5$ \\
\hline $60 \mathrm{~h}$ & 52 & $46(88)$ & $30(58)^{\mathrm{ab}}$ & $30(58)$ & $79 \pm 4$ \\
\hline
\end{tabular}

a,bSignificantly different $(P<0.05$, Chi square); pi, post insemination.

Gonadotrophins (FSH and LH) are widely used as supplements in maturation media and their action on cumulus expansion, meiotic resumption and cytoplasmic maturation has been shown in different systems (reviewed by Gordon, 1994). However, effects of gonadotrophins in vitro remain controversial in cattle, possibly due to the diversity of the preparations and of the concentrations used by different workers (reviewed by Bevers et al., 1997). It is still not clear whether gonadotrophins have direct effects on cumulus and oocyte, or potentiate the action of growth factors present in follicular fluid (review by Gandolfi, 1996).

The LH peak triggers a change in the steroid content of follicular fluid. Consequently, the meiotic process occurs in a changing steroid environment with a progressive decrease in oestradiol concentration and increase in progesterone. However, the possible effect of steroids on meiotic resumption or on cytoplasmic maturation remains controversial in domestic mammals (review by Bevers et al., 1997), although oestradiol is frequently added to maturation media. Oestradiol has been shown to increase the developmental potential of maturing human oocytes without affecting meiotic progression (Tesarik and Mendoza, 1995). In this case, oestradiol acts directly at the plasma membrane (non-genomic effect) by allowing influx of calcium ions, initiating several calcium oscillations in the ooplasm.

Some recent data support the possible intervention of growth factors, hormones and intraovarian peptides (review by Bevers et al., 1997). The effect of epidermal growth factor has been investigated in numerous species including some domestic ruminants. A role for EGF in both cytoplasmic and nuclear oocyte maturation as well as in development of the preimplantation embryo has been identified in cattle (Lorenzo et al., 1995; Lonergan et al., 1996). The addition of $10 \mathrm{ng}$ EGF $\mathrm{ml}^{-1}$ to M199 medium resulted in increased rates of development that were similar to the addition of $10 \%$ fetal calf serum. The presence of EGF in follicular fluid as well as intrafollicular 
EGF-binding sites have been reported in numerous species and the expression of this factor and of its receptor seems to be under the control of both gonadotrophins and steroids (see Lonergan et al., 1996 for references). Taken together, these observations indicate a central function for EGF or related molecules in the mediation of intrafollicular regulation of oocyte capacitation and maturation.

The insulin-like growth factors (IGF-I and IGF-II) and related proteins (IGF-binding proteins and IGFBP-specific proteases) are suspected to play a major role in intra-ovarian regulation of follicular growth and differentiation (review by Monniaux et al., 1997). The action of IGFs on oocyte maturation has been widely investigated but remains controversial and IGF activity seems to be dependent on the presence of other factors. Growth hormone $(\mathrm{GH})$ is known to improve the superovulatory response in cattle. GH is thought to exert its action by regulating expression of IGFs in various organs including the ovary. $\mathrm{GH}$ has recently been shown to accelerate oocyte maturation in cattle and to increase the developmental potential of mature oocytes (Izadyar et al., 1996). However, this effect is not mediated by IGFs since the effect of GH can be obtained in the presence of anti-IGF-I antibody. Furthermore, bovine oocytes as well as cumulus cells express GH receptor and, consequently, are able to respond directly to GH stimulation (Izadyar et al., 1997). A positive effect of the inhibin-activin family of ovarian glycoproteins on oocyte developmental competence has been reported recently in cattle (Stock et al., 1997). Human recombinant inhibin A and activin A added to defined IVM medium enhanced the development of cleaved bovine embryos to the blastocyst stage alone $\left(10 \mathrm{ng} \mathrm{ml}^{-1}\right)$ or in combination $\left(1 \mathrm{ng} \mathrm{ml}^{-1}\right.$ of each) without affecting the cleavage rate. Thus inhibin and activin appear to increase the quality of oocyte cytoplasmic maturation. This effect could be mediated through an action on meiotic resumption and progression since nuclear maturation was advanced by the addition of activin, inhibin or both factors, whereas the final metaphase II rate was not affected. A possible link between the kinetics of progression of meiotic events and the resulting developmental ability of the oocytes has already been reported in cattle (Dominko and First, 1997), indicating that these two events (nuclear and cytoplasmic maturation) are strongly correlated and might be regulated by common factors such as the activin-inhibin system. Inhibin and activin may act directly on oocytes, because their action does not involve cumulus expansion (Stock et al., 1997) and because they are able to stimulate the cytoplasmic maturation of denuded oocytes (Silva and Knight, 1998). In addition, the expression of the activin receptor has been detected by RT-PCR in oocytes as well as in cumulus cells (Izadyar et al., 1998). Inhibin and activin are present in follicular fluid and their concentration is increased by the time of meiotic resumption in pig follicles (Miller et al., 1991), indicating that they might be involved in the regulation of meiosis.

To summarize, oocyte maturation includes nuclear (meiotic resumption and progression from prophase I to metaphase II) and cytoplasmic events. Nuclear maturation is spontaneous when the follicular inhibitory signal is suppressed (LH surge or in vitro culture). However, some oocytes need stimulation to complete meiosis. More oocytes are dependent upon external signalling for completion of cytoplasmic maturation. EGF seems to be one of the most potent factors stimulating both aspects of maturation, but other factors (GH, activin, inhibin) contained in follicular fluid may also regulate the different aspects of oocyte maturation.

\section{Criteria of Oocyte Quality}

Some of the events related to oocyte capacitation and maturation are known. However, we still lack knowledge of real markers strictly linked to the final developmental competence of the oocyte, except the observation of their development after IVF. Such markers would be helpful in investigating the conditions of in vitro maturation or to test treatments on animals to increase the occurrence of follicles containing competent oocytes. It would therefore be of interest to determine some morphological or biochemical characteristics of highly competent oocytes, of their surrounding cells and of follicles containing such oocytes.

The first visible characteristic that might be linked to the developmental competence is the morphology of the cumulus-oocyte complex (COC) obtained after follicular aspiration. Most of the IVP laboratories use the same morphological evaluation and selection of the COCs entering IVM 
based on the appearance of the cumulus (several layers, continuous, compact) and of the ooplasm (Leibfried and First, 1979). However, this morphological evaluation is not sufficient to predict the developmental potential of a given oocyte and the identification of markers more strictly linked to this potential would be helpful.

The discovery of such markers of oocyte quality requires some experimental models providing highly and poorly competent oocytes in a reproducible way. Some follicular characteristics such as follicular diameter or stage of atresia could provide comparative models of developmental competence as could some genotype particularities in sheep, such as the Booroola gene (see above). In addition, oocytes originated from prepubertal animals provide negative models of developmental competence in cattle (Revel et al., 1995; Khatir et al., 1996), sheep (O'Brien et al., 1997) and goat (Martino et al., 1994). These oocytes can resume meiosis, reach metaphase II, be fertilized and cleave in the same proportion as their adult counterparts, but their ability to develop to the blastocyst stage is strongly reduced. The same discrepancy was observed when nuclear transfer was used instead of IVF in cattle, underlining the cytoplasmic origin of the deficiency of prepubertal oocytes (Mermillod et al., 1998).

The differential analysis of oocytes from these models with different approaches (such as morphology, ultrastructure, protein synthesis and phosphorylation and expression of receptors) has already provided some clues toward a better comprehension of oocyte quality and will certainly allow the identification of some markers of oocyte quality that will help to improve in vitro embryo production in future.

\section{Conclusion}

In conclusion, it is clear that oocyte quality is decisive for the success of in vitro production of embryos from domestic ruminants. Oocyte quality is the consequence of both oocyte capacitation during the course of folliculogenesis and oocyte maturation after the LH surge in vivo or after in vitro culture during IVM. An appropriate follicular environment is essential to facilitate these two aspects of oocyte differentiation.

\section{References}

Arlotto T, Schwartz I-L, First NL and Leibfried-Rutledge ML (1996) Aspects of follicle and oocyte stage that affect in vitro maturation and development of bovine oocytes Theriogenology $45943-956$

Bevers MM, Dieleman SJ, van den Hurk R and Izadyar F (1997) Regulation and modulation of oocyte maturation in the bovine Theriogenology 47 13-22

Bindon BM (1984) Reproductive biology of the Booroola Merino sheep Australian Joumal of Biological Science 37 163-189

Blondin P and Sirard MA (1995) Oocyte and follicular morphology as determining characteristics for developmental competence in bovine oocytes Molccular Reproduction and Development $4154-62$

Campbell KHS and Wilmut I (1997) Totipotency and multipotentiality of cultured cellst applications and progress Theriogenology $4763-72$

Carolan C, Lonergan P, Khatir H and Mermillod P (1996a) The in vitro production of bovine embryos using individual oocytes Molecular Reproduction and Development 45 145-154

Carolan C, Lonergan P, Monget P, Monniaux D and Mermillod P (1996b) Effect of follicle size and quality on the ability of follicular fluid to support cytoplasmic maturation of bovine oocytes Molecular Reproduction and Development 43 $477-483$
Cognié Y, Poulin N, Pisselet C and Monniaux D (1995) Effect of atresia on the ability of follicular fluid to support cytoplasmic maturation of sheep oocytes in vitro. Theriogenology 43188 (Abstract)

Cogniě Y, Benoit F, Poulin N, Khatir H and Driancourt MA (1998) Effect of follicle size and of the $\mathrm{Fec} B$ booroola gene on oocyte function in sheep fournal of Reproduction and Ferfility 112 379-386

Crozet N, Ahmed-Ali M and Dubos MP (1995) Developmental competence of goat oocytes from follicles of different size categories following maturation, fertilization and culture in vitro. Tournal of Reproduction and Fertility 103 293-298

Dominko T and First NL (1997) Timing of meiotic progression in bovine oocytes and its effect on early embryo development Molecular Reproduction and Development 47 456-467

Downs SM (1996) Regulation of meiotic arrest and resumption in mammalian oocytes. In The Ovary: Regulation, Dysfunction And Treatment pp 141-148 Ed. M Filicori and C. Flamigni. Elsevier, Amsterdam

Driancourt MA, Thuel B, Mermillod P and Lonergan P (1998) Relationship between oocyte quality (measured after IVM) IVF and IVC of individual oocytes) and follicle function in cattle Theriogenology 49345 (Abstract) 
Ectors FJ, Koulischer $\mathrm{L}_{r}$ Jamar M, Herens C, Verloes A, Remy B and Beckers JF (1995) Cytogenetic study of bovine oocytes matured in vitro. Theriogenology $44445-450$

Eppig JJ (1996) Coordination of nuclear and cytoplasmic oocyte maturation in eutherian mammals Reproduction Fertility and Development 8 485-489

Eppig JJ, O'Brien M and Wiggleworth K (1996) Mammalian oocyte growth and development in vitro. Molecular Reproduction and Development 44 260-273

Ferry L, Mermillod P, Massip A and Dessy F (1994) Bovine embryos cultured in serum-poor conditioned medium need cooperation to reach the blastocyst stage Theriogenology 42 445-453

Gandolfi F (1996) Intra-ovarian regulation of oocyte developmental competence in cattle Zygote 4 323-326

Gordon I (1994) Laboratory Production of Cattle Embryos CAB International, Oxon

Gosden R, Krapez J and Briggs D (1997) Growth and development of the mammalian oocyte BioEssays 19 875-882

Grime RW and Ireland JJ (1986) Relationship of macroscopic appearance of the surface of bovine ovarian follicles, concentrations of steroids in follicular fluid, and maturation of oocytes in vitro. Biology of Reproduction 35 $725-732$

Guilbault LA, Rouillier P, Matton P, Glencross RG, Beard AJ and Knight PG (1993) Relationships between the level of atresia and inhibin contents (alpha subunit and ab dimer) in morphologically dominant follicles during their growing and regressing phases of development in cattle Biology of Reproduction 48 268-276

Harada M, Miyano T, Matsumura K, Osaki S, Miyake M and Kato S (1997) Bovine oocytes from early antral follicles grow to meiotic competence in vitro: effect of FSH and hypoxanthine Theriogenology $78743-755$

Hazeleger NL, Hill DJ, Stubbings RB and Walton JS (1995) Relationship of morphology and follicular fluid environment of bovine oocytes to their developmental potential in vitro. Theriogenology 43 509-522

Hyttel P, Fair T, Callesen H and Greve T (1997) Oocyte growth, capacitation and final maturation in cattle Theriogenology $4723-32$

Izadyar F, Colenbrander B and Bevers MM (1996) In vitro maturation of bovine oocytes in the presence of growth hormone accelerates nuclear maturation and promotes subsequent embryonic development Molecular Reproduction and Development 45 372-377

Izadyar F, Van Tol HTA, Colenbrander B and Bevers MM (1997) Stimulatory effect of growth hormone on in vitro maturation of bovine oocytes is exerted through cumulus cells and not mediated by IGF-I Molecular Reproduction and Development 47 175-180

Izadyar F, Colenbrander B and Bevers MM (1998) Stimulatory effect of growth hormone on in vitro maturation of bovine oocytes is exerted through the cyclic adenosine $3^{\prime}, 5^{\prime}$ monophosphate signaling pathway Biology of Reproduction 57 1484-1489

Khatir H, Lonergan P, Carolan C and Mermillod P (1996) The prepubertal oocytes as a negative model in the study of bovine oocyte developmental competence acquisition Molecular Reproduction and Development 45 231-239

Kubota C and Yang X (1998) Cytoplasmic incompetence results in poor development of bovine oocytes derived from small follicles Theriogenology 47183 (Abstract)
Leibfried L and First NL (1979) Characterization of bovine follicular oocytes and their ability to mature in vitro. Journal of Animal Science 48 76-86

Lonergan P, Monaghan P, Rizos D, Boland MP and Gordon I (1994a) Effect of follicle size on bovine oocyte quality and developmental competence following maturation, fertilization, and culture in vitro. Molecular Reproduction and Development $3748-53$

Lonergan P, Carolan C and Mermillod P (1994b) Development of bovine embryos in vitro following oocyte maturation under defined conditions Reproduction, Nutrition and Development 34 329-339

Lonergan P, Carolan C, Van Langendonckt A, Donnay I, Khatir H and Mermillod P (1996) Role of epidermal growth factor in bovine oocyte maturation and preimplantation embryo development Biology of Reproduction 54 1412-1421

Lonergan P, Khatir H, Carolan C and Mermillod P (1997) Bovine blastocyst production in vitro following inhibition of oocyte meiotic resumption for $24 \mathrm{~h}$ Journal of Reproduction and Fertility 109 355-365

Lorenzo PL, Illera MJ, Illera JC and Illera M (1995) Role of EGF, IGF-I, sera and cumulus cells on maturation in vitro of bovine oocytes Theriogenology 44 109-118

Martino A, Mogas T, Palomo MJ and Paramio MT (1994) Meiotic competence of prepubertal goat oocytes Theriogenology $4 \mathbf{1}$ 969-980

Massip A, Mermillod P and Dinnyes A (1995) Morphology and biochemistry of in-vitro produced bovine embryos: implications for their cryopreservation Human Reproduction 10 3004-3011

Mermillod P, Lonergan P, Carolan C, Khatir H, Poulin N and Cognié Y (1996) Oocyte maturation in domestic ruminants Contraception Fertilité Sexualité 24 552-558

Mermillod P, Peynot N, Lonergan P, Khatir H, Driancourt MA, Renard JP and Heyman Y (1998) Developmental potential of oocytes collected from 8-15 day old unstimulated or FSH treated calves Theriogenology 47294 (Abstract)

Miller KF, Xie S and Pope W (1991) Immunoreactive inhibin in follicular fluid is related to meiotic stage of the oocyte during final maturation of the porcine follicle Molecular Reproduction and Development 28 35-39

Monget $\mathrm{P}$, Monniaux D, Pisselet C and Durand P (1993) Changes in insulin-like growth factor-I (IGF-I), IGF-II, and their binding proteins during growth and atresia of ovine ovarian follicles Endocrinology 132 1438-1446

Monniaux D, Huet C, Besnard N, Clément F, Bosc M, Pisselet C, Monget P and Mariana JC (1997) Follicular growth and ovarian dynamics in mammals Journal of Reproduction and Fertility Supplement 51 3-23

Moor RM, Lee C, Dai YF and Fulka J, Jr (1996) Antral follicles confer developmental competence on oocytes Zygote 4 289-293

Nuttinck F, Mermillod P, Massip A and Dessy F (1993) Characterization of in vitro growth of bovine preantral ovarian follicles: a preliminary study Theriogenology 39 811-821

O'Brien JK, Catt SL, Ireland KA, Maxwell WMC and Evans G (1997) In vitro and in vivo developmental capacity of oocytes from prepubertal and adult sheep Theriogenology 47 1433-1443

Pavlok A, Lucas-Hahn A and Niemann H (1992) Fertilization and developmental competence of bovine oocytes derived from different categories of antral follicles Molecular Reproduction and Development 31 63-67 
Revel F, Mermillod P, Peynot N, Renard JP and Heyman Y (1995) Comparison of developmental ability of oocytes from prepubertal calves and adult cows Journal of Reproduction and Fertility 103 115-120

Silva CC and Knight PG (1998) Modulatory actions of activin-A and follistatin on the developmental competence of in vitromatured bovine oocytes Biology of Reproduction 58 558-565

Sirard MA and Blondin P (1996) Oocyte maturation and IVF in cattle Animal Reproduction Science 42 417-426

Sirard MA, Roy F, Mermillod P and Guilbault LA (1995) The origin of follicular fluid added to the media during bovine IVM influences embryonic development Theriogenology 44 85-94

Stock AE, Woodruff TK and Smith LC (1997) Effects of inhibin A and activin A during in vitro maturation of bovine oocytes in hormone- and serum-free medium Biology of Reproduction 56 1559-1564

Taieb F, Thibier C and Jessus C (1997) On cyclins, oocytes, and eggs Molecular Reproduction and Development 48 397-411

Tesarik J and Mendoza C (1995) Nongenomic effects of 17 beta- estradiol on maturing human oocytes: relationship to oocyte developmental potential Journal of Clinical Endocrinology and Metabolism 80 1438-1443

Thibier M and Saumande J (1975) Oestradiol-17ß, progesterone and 17alpha-hydroxyprogesterone concentration in jugular venous plasma in cows prior to and during oestrus Journal of Steroid Biochemistry 6 1433-1437

Thompson JG (1996) Defining the requirements for bovine embryo culture Theriogenology $4527-40$

Thompson JG (1997) Comparison between in vivo-derived and in vitro-produced pre-elongation embryos from domestic ruminants Reproduction Fertility and Development 9 341-354

Van Tol HTA, Van Eijk MJT, Mummery CL, Van Den Hurk R and Bevers MM (1996) Influence of FSH and hCG on the resumption of meiosis of bovine oocytes surrounded by cumulus cells connected to membrana granulosa Molecular Reproduction and Development 45 218-224

Wassarman PM and Albertini DF (1994) The mammalian ovum. In Physiology of Reproduction pp A79-A122 Ed. E Knobil and JD Neill. Raven Press, New York 